\title{
Lessons from the Past: Comparison of the Disease Burden of the Influenza A (H1N1) Pandemic 2009- 10 and Seasonal Influenza 2010-2019 in the United States
}

James A Koziol ( $\sim$ jkoziol@prism-sd.org)

Proteogenomics Research Institute for Systems Medicine

\section{Research Article}

Keywords: Influenza, Pandemic, H1N1

Posted Date: February 17th, 2021

DOl: https://doi.org/10.21203/rs.3.rs-198743/v1

License: (9) This work is licensed under a Creative Commons Attribution 4.0 International License. Read Full License 


\section{Abstract \\ Background}

Annual influenza outbreaks constitute a major public health concern, both in the United States and worldwide. Comparisons of the health burdens of outbreaks might lead to the identification of specific atrisk populations, for whom public health resources should be marshaled appropriately and equitably.

\section{Methods}

We examined the disease burden of the 2009-10 influenza A (H1N1) pandemic relating to illnesses, medical visits, hospitalizations, and mortality, compared to influenza seasons 2010 to 2019, in the United States, as compiled by the Centers for Disease Control.

\section{Results}

With regard to seasonal influenza, rates of illnesses and medical visits were highest in infants aged 0-4 years, followed by adults aged 50-64 years. Rates of hospitalizations and deaths evinced a starkly different pattern, both dominated by elderly adults aged 65 and over. Youths aged 0 to 17 years were especially adversely affected by the H1N1 pandemic relative to hospitalizations and mortality compared to seasonal influenza; but curiously the opposite pattern was observed in elderly adults (aged 65 and older).

\section{Conclusions}

The disease burden of the 2009-10 influenza A pandemic was strikingly unlike that observed in the subsequent influenza seasons 2010 to 2019, in the United States: the past did not predict the future.

\section{Background}

A reasonable approach to control or mitigate the adverse impact of seasonal influenza and pandemics in the United States would be to focus resources on those subsets of the population most heavily affected by the disease. To that end, we summarize the burden of disease as reflected by illnesses, hospitalizations and mortality associated with the 2009-10 influenza A (H1N1) pandemic, compared to the subsequent influenza seasons 2010 to 2019, as compiled by the Centers for Disease Control. Our goal is to identify specific at-risk populations, for whom public health resources should be marshaled appropriately and equitably.

\section{Methods}


We utilized tables prepared by the Centers for Disease Control (CDC) relating to the estimated influenza disease burden in the United States, from the 2010-11 through the 2019-2020 influenza seasons. ${ }^{1}$ Each seasonal table contains estimates of the numbers of symptomatic illnesses, medical visits, hospitalizations, and deaths attributable to influenza, by age groups $0-4$ years, 5-17 years, 18-64 years, and $65+$ years, with associated estimated ranges ${ }^{2,3}$. In addition, estimated rates of these influenza disease outcomes per 100,000 population in each of these age groups are also provided.

We obtained data relating to the impact of the influenza A (H1N1) pandemic in the United States during the period April 2009 through April 2010 from CDC investigators. ${ }^{4}$ These data consist of estimates of illnesses, hospitalizations, and deaths attributable to influenza A, along with ranges, by age groups $0-17$ years, 18-64 years, and 65 + years. We converted these counts into rates (per 100000) using 2010 population figures from the US Census Bureau ${ }^{5}$. We remark that the CDC has implemented rigorous protocols $^{1-4}$ for data tabulation and presentation, furthering credence in their reports.

To ensure comparability between the influenza A (H1N1) data from 2009-10 and observations in subsequent years, we pooled the age groups $0-4$ and $5-17$ years in the CDC annual tabulations, and used a random effects beta binomial model ${ }^{2,6}$ with linear time trend $d^{7,8}$ to summarize the weighted average (consensus) disease burden over the period 2010-2020 relating to rates of illnesses, hospitalizations, and deaths for the age groups 0-17 years, 18-64 years, and 65+years. Calculations were performed in Stata v.14 (StataCorp, College Station, Texas, 2015), as outlined by Guimaraes ${ }^{9}$.

\section{Results}

In Fig. 1, we depict the rates of illnesses, medical visits, hospitalizations, and mortality across the age groups $0-4,5-17,18-64$, and $65+$ attributable to influenza, from the CDC compilations. Rates of illnesses and medical visits are highest in infants age 0-4 years, followed by adults age 50-64 years. Rates of hospitalizations and deaths evince a starkly different pattern, both dominated by elderly adults age 65 and over.

We show the age-specific rates of illnesses, hospitalizations, and deaths in Fig. 2, along with summary measures of the rates over 2010-19, from a beta binomial random effects model. We also depict the corresponding rates from the 2009-2010 influenza A (H1N1) pandemic ${ }^{4}$. Estimated ranges are also shown for the yearly age-specific rates, and $99 \%$ confidence intervals are given for the summary consensus rates.

Lastly, we abstracted in Fig. 3 the rates and ranges from Fig. 2 corresponding to the 2009-10 influenza A pandemic, the consensus values for 2010-19, and the individual rates from 2011-12 (a "low impact" flu season, from Fig. 1) and 2017-18 (a "high impact" flu season, from Fig. 1), to facilitate comparisons across different age groups.

\section{Discussion}


Patterns in the annual rates of illnesses, medical visits, hospitalizations, and deaths attributable to seasonal influenza according to age are revealing (Fig. 1). Clearly, the overall health impact varies by year, but relative differences between age groups are fairly stable. Rates of illnesses and medical visits are highest in infants age $0-4$ years, followed by adults age 50-64 years. One might conjecture that infants naive to novel influenza viruses would be highly susceptible to illness, but recovery would typically be expected. Rates of hospitalizations and deaths evince a starkly different pattern, both dominated by elderly adults age 65 and over. Vulnerability in this age group is probably exacerbated by pre-existing health conditions, so avoidance of initial infection would be a prudent strategy.

How does the influenza A (H1N1) pandemic of 2009-2010 compare to the subsequent "normal" influenza seasons? Rates of illnesses during the H1N1 pandemic (2009-10) were about 2.4 times greater than the consensus rates for seasonal influenza (2010-19) across all age groups. For youths aged 0 to 17 , the $\mathrm{H} 1 \mathrm{~N} 1$ hospitalization rate was about 2.6 times greater than the consensus rate for seasonal influenza, and the mortality rate was 2.9 times higher. One might thereby infer that influenza $A(\mathrm{H} 1 \mathrm{~N} 1)$ is intrinsically more severe among youths than seasonal influenza. Among adults aged 18 to 64 , hospitalization rates and mortality rates from $\mathrm{H} 1 \mathrm{~N} 1$ were 1.3 and 1.4 times greater respectively than the corresponding consensus rates for seasonal influenza. On the other hand, the hospitalization and mortality rates for elderly adults (aged 65 and older) during the H1N1 pandemic appear anomalous at a mere one-tenth the corresponding consensus rates for seasonal influenza, and their corresponding ranges seem disproportionately small. In light of subsequent findings, we conjecture that hospitalizations and deaths (and their spread) in this age cohort during the H1N1 pandemic were underestimated; in particular, one might expect that the hospitalization and mortality rates would be higher than in the younger adults aged 18 to 64 . It is therefore doubtful that the influenza A pandemic of 2009-10 is a suitable model for subsequent influenza epidemics relative to the experience of elderly adults.

An important limitation of this retrospective analysis is the absence of information on other potential population characteristics and risk factors, such as gender and comorbidities, which likely affect disease morbidity and mortality. And, as noted above, possible under-detection of morbidity and mortality during the H1N1 pandemic in elderly adults might lead to erroneous inferences in highlighting specific at-risk populations or focusing preventive measures toward them.

\section{Conclusions}

The disease burden of the 2009-10 influenza A pandemic was strikingly unlike that observed in the subsequent influenza seasons 2010 to 2019, in the United States, though questions remain concerning the validity of some of the 2009-10 pandemic findings.

\section{Declarations}

\section{Availability of data and materials}


The data relating to the influenza burden of disease for the years 2010-2019 in the United States are freely available on the Centers for Disease Control website, https://www.cdc.gov/flu/about/burden/index.html.

The data relating to the 2009-10 influenza A pandemic burden of disease uis also freely available, and were taken directly from reference \#4.

\section{Ethics approval and consent to participate}

Not Applicable

\section{Consent for publication}

Not Applicable

\section{Competing interests}

The author declares no competing interests.

\section{Funding}

This research was supported by grant P01 HL119165, from the National Institutes of Health.

\section{Authors' contributions}

JK designed, this study, undertook all analyses, and wrote the manuscript.

\section{References}

1. Estimated influenza disease burden by season, 2010-11 through 2019-20 influenza seasons. Centers for Disease Control website. Accessed December 1, 2010. https://www.cdc.gov/flu/about/burden/index.html

2. Reed C, Chaves SS, Kirley PD et al. Estimating influenza disease burden from population-based surveillance data in the United States. PLoS ONE 2015;10:e0118369. doi:10.1371/journal.pone.0118369

3. Rolfes, MA, Foppa, IM, Garg, S, et al. Annual estimates of the burden of seasonal influenza in the United States: A tool for strengthening influenza surveillance and preparedness. Influenza Other Respir Viruses. 2018;12:132-137. https://doi.org/10.1111/irv.12486

4. Shrestha SS, Swerdlow DL, Borse RH et al. Estimating the burden of 2009 pandemic influenza A (H1N1) in the United States (April 2009-April 2010). Clin Infect Dis 2011;52 Suppl 1:S75-82. doi: 10.1093/cid/ciq012.

5. Howden LM, Meyer JA. Age and sex composition: 2010. 2010 Census Briefs. Publication C2010BR03, US Census Bureau, May 2011. https://www.census.gov/prod/cen2010/briefs/c2010br-03.pdf 
6. Young-Xu Y, Chan KA. Pooling overdispersed binomial data to estimate event rate. BMC Medical Research Methodology 2008;8:58. doi:10.1186/1471-2288-8-58.

7. Woolf SH, Chapman DA, Sabo RT, Weinberger DM, Hill L. Excess deaths from COVID-19 and other causes, March-April 2020. JAMA 2020;324:510-513. doi:10.1001/jama.2020.11787

8. Woolf SH, Chapman DA, Sabo RT, Weinberger DM, Hill L. Excess deaths from COVID-19 and other causes, March-July 2020. JAMA 2020;324:1562-1564. doi:10.1001/jama.2020.19545

9. Guimarães P. A simple approach to fit the beta-binomial model. Stata Journal 2005;5:385-394.

\section{Figures}
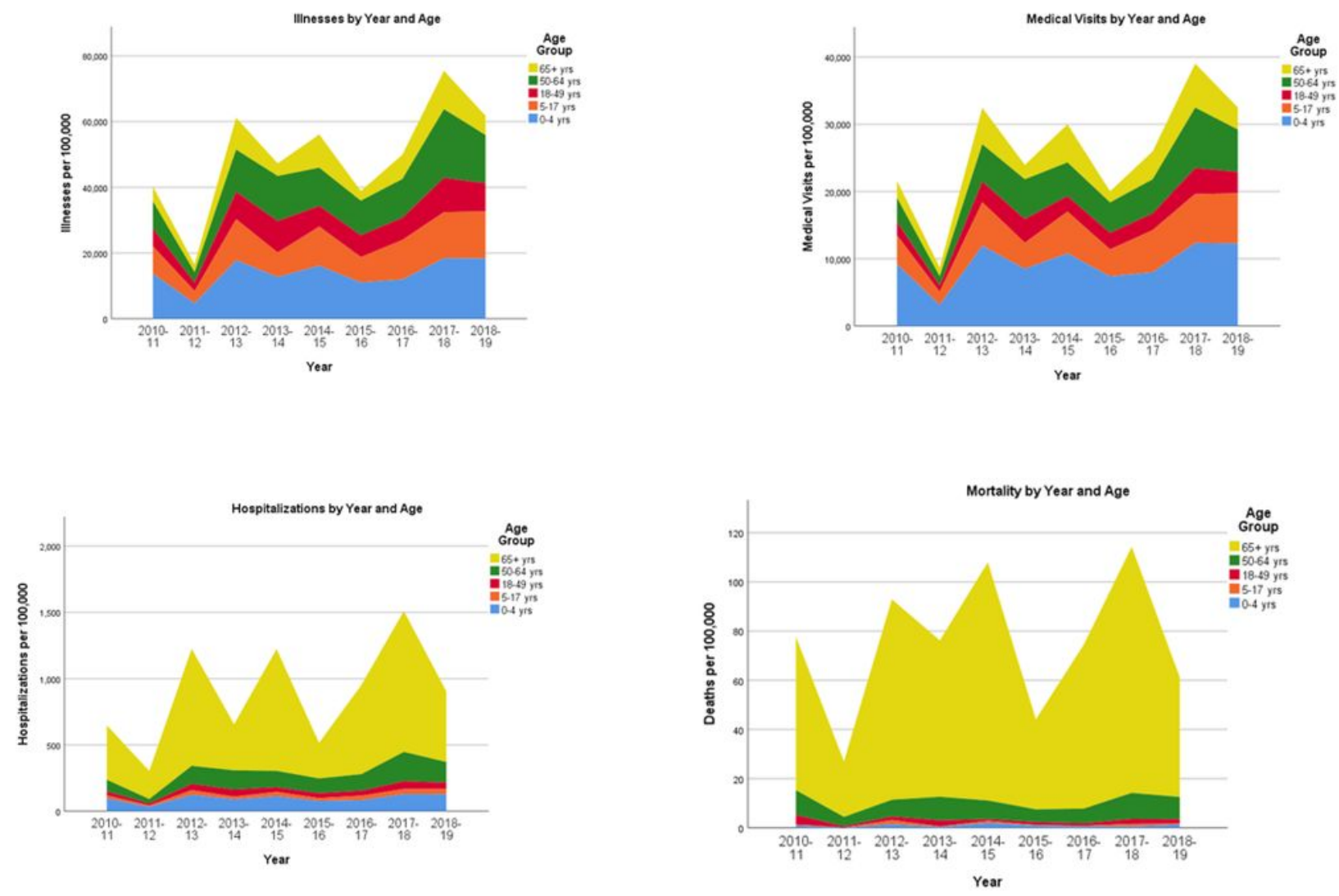

\section{Figure 1}

Stacked histograms of illnesses (Figure 1A), medical visits (Figure 1B), hospitalizations (Figure 1C), and deaths (Figure 1D) per 100000 population, by year and age group, 2010-19. Annual observed counts are taken from the Centers for Disease Control website1, for the entire United States. 

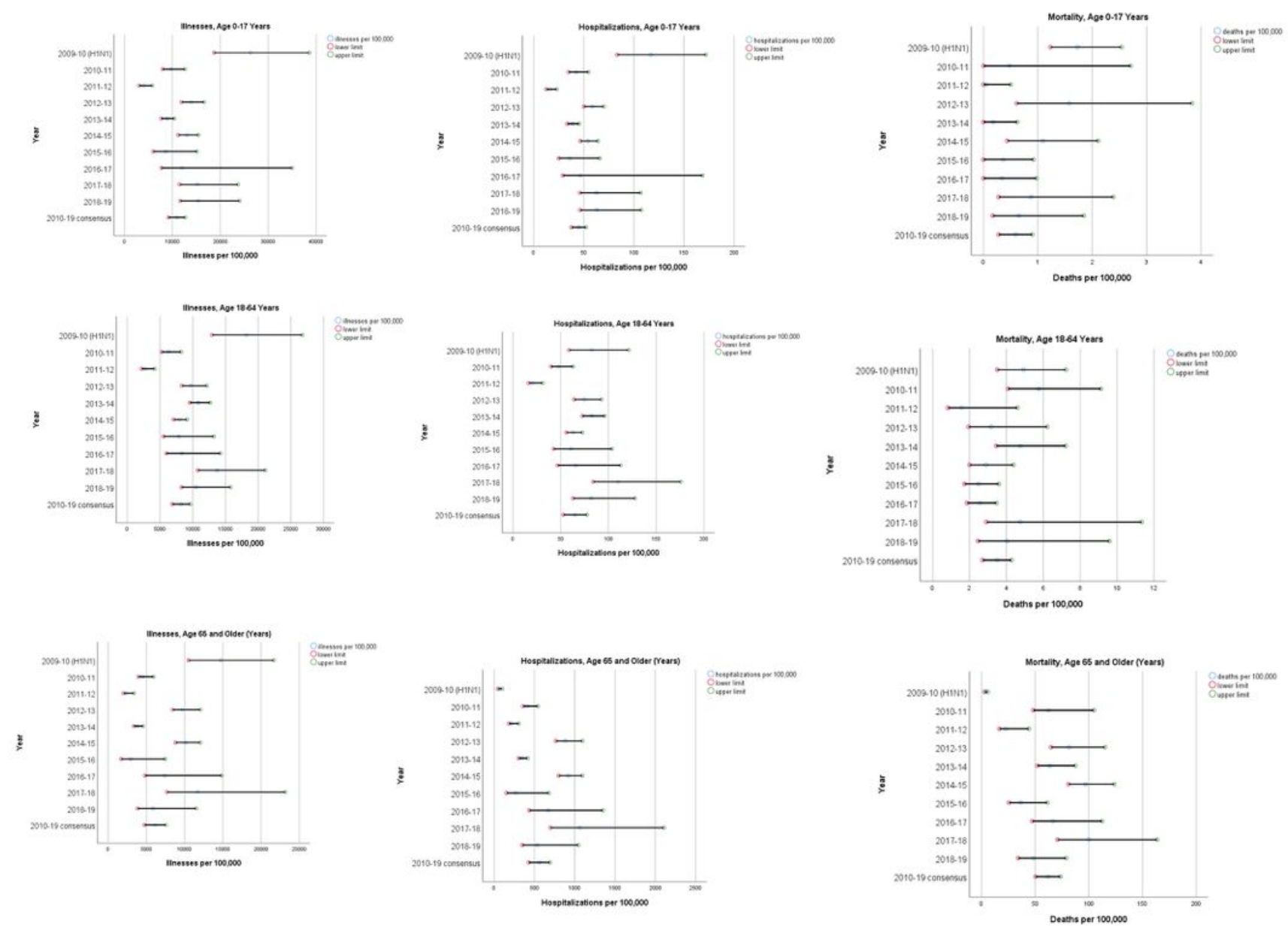

Figure 2

Rates of illnesses, hospitalizations, and deaths attributable to the influenza A (H1N1) pandemic (200910)4 and seasonal influenza (2010-19)1, by age group (A-C:0-17 years; D-F: 18-64 years; G-l: 65+ years). The lower and upper limits represent estimated ranges for the 2009-10 through 2018-19 years. The lower and upper limits for the 2010-19 consensus values are 99\% confidence intervals for the overall estimate, determined from a beta-binomial regression model2,11,12 for the 2010-11 through 2018-19 years.
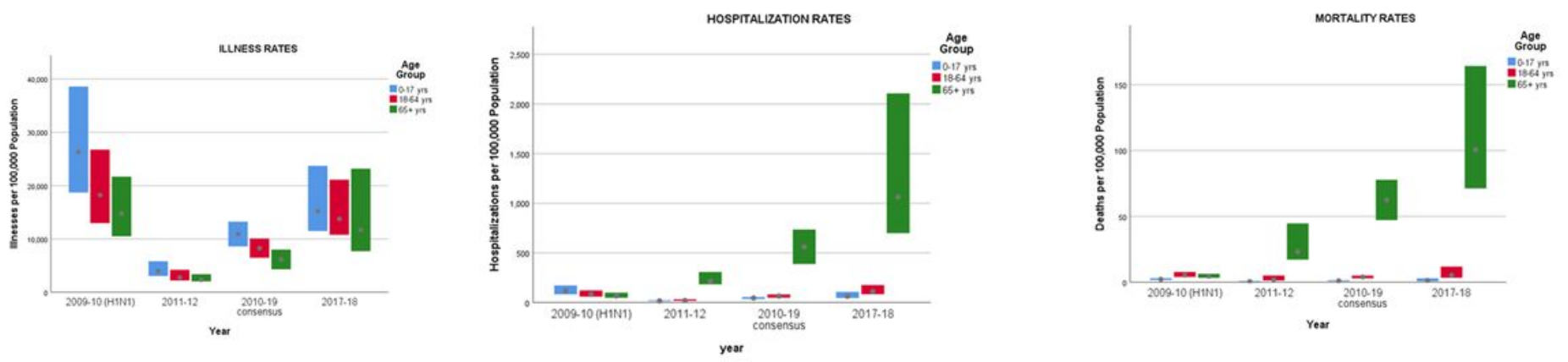


\section{Figure 3}

Range charts of rates of illnesses (A), hospitalizations (B), and deaths (C) attributable to the influenza A (H1N1) pandemic (2009-10), seasonal influenza for 2011-12 and 2017-18, and consensus values for 2010-19, by age group (0-17 years, 18-64 years, and 65+ years). 